\title{
Recommendations for Pregnant Members of Dermatology Health Care Teams During the COVID-19 Pandemic
}

\author{
Jose W. Ricardo, MD; Shari R. Lipner, MD, PhD
}

\section{PRACTICE POINTS}

- Pregnant women are at an increased risk for severe illness due to COVID-19 compared with nonpregnant women; therefore, it is important to protect pregnant health care workers who are caring for patients during the current pandemic.

- Although currently available COVID-19 vaccines have not been tested in pregnant women, they should not be withheld from pregnant individuals.

- Pregnant attending physicians and residents in dermatology can continue to provide care through telemedicine; if they choose to, and if all recommended personal protective equipment (PPE) are available, they can continue to provide in-person care.

- Correct and comprehensive use of PPE by pregnant health care workers is paramount to minimizing exposure to SARS-CoV-2.

nformation is scarce regarding the impact of COVID-19 on pregnant women and newborns; health care workers (HCWs), particularly pregnant women, ${ }^{1}$ who are caring for patients during the pandemic might experience concern and uncertainty. The American College of Obstetricians and Gynecologists (ACOG) released recommendations, based on expert consensus, regarding pregnant HCWs on December 14, 2020. ${ }^{2}$ We propose an appropriation of the ACOG recommendations for dermatologists and their teams caring for patients during the COVID-19 pandemic.

\section{Risks to Pregnant HCWs}

Worldwide, viral pneumonia is a leading cause of death during pregnancy, ${ }^{3}$ with higher mortality documented among pregnant patients during the 1918 influenza pandemic and the 2003 severe acute respiratory syndromeassociated coronavirus pandemic, ${ }^{3}$ and an increased rate of hospital admission documented among these patients compared to the general population during the 2009 H1N1 influenza pandemic. ${ }^{4}$

Data from the Centers for Disease Control and Prevention (CDC) suggest that pregnant women with symptomatic COVID-19 $(n=30,415)$ are at increased risk for the following (compared to nonpregnant women with symptomatic COVID-19 [n=431,410] $)^{5}$ :

- Admission to the intensive care unit (10.5 of every 1000 cases vs 3.9 of every 1000 cases; adjusted risk ratio $[\mathrm{aRR}]=3.0 ; 95 \% \mathrm{CI}, 2.6-3.4)$

- Receipt of invasive ventilation (2.9 of every 1000 cases vs 1.1 of every 1000 cases; aRR $=2.9$; 95\% CI, 2.2-3.8)

- Receipt of extracorporeal membrane oxygenation (0.7 of every 1000 cases vs 0.3 of every 1000 cases; $\mathrm{aRR}=2.4 ; 95 \% \mathrm{CI}, 1.5-4.0)$

- Death (1.5 of every 1000 cases vs 1.2 of every 1000 cases; $\mathrm{aRR}=1.7 ; 95 \% \mathrm{CI}, 1.2-2.4$ ).

Although the absolute risk of severe COVID-19related outcomes is low, the CDC includes pregnant women in its increased risk category for COVID-19. Furthermore, in a systematic review of 61 studies comprising 790 COVID-19-positive pregnant women and 548 newborns, the rates of cesarean delivery, premature birth, low birth weight, and adverse pregnancy events

From the Department of Dermatology, Weill Cornell Medicine, New York, New York

The authors report no conflict of interest.

Correspondence: Shari R. Lipner MD, PhD, 1305 York Ave, New York, NY 10021 (shl9032@med.cornell.edu).

doi:10.12788/cutis.0250 
(the latter comprising preterm birth, death or stillbirth, and early termination of pregnancy) were estimated to be $72 \%, 23 \%, 7 \%$, and $27 \%$, respectively. ${ }^{6}$ In a systematic review of 39 studies (case series and cohort studies), comprising 936 SARS-CoV-2-tested newborns of mothers with COVID-19, mother-to-fetus transmission of SARS-CoV-2 occurred during the third trimester in approximately $3.2 \%$ of infected mothers. ${ }^{7}$

In pregnant women with COVID-19 who develop cytokine storm syndrome, a fetal inflammatory response syndrome can ensue, which has been shown to cause ventricular expansion and bleeding in animal models. ${ }^{8}$ In addition, underlying conditions, such as cardiovascular disease, diabetes mellitus, pre-existing lung disease, and obesity, which are well-established risks factors for severe COVID-19 in nonpregnant patients, can increase the severity of COVID-19 in pregnant women. . $^{5,-11}$

\section{Recommendations From ACOG for Pregnant HCWs}

The American College of Obstetricians and Gynecologists recommends that health care facilities consider limiting the exposure of pregnant HCWs to patients with confirmed or suspected COVID-19. They also recommend that pregnant women continue to work in patient-facing roles if they want to, if recommended personal protective equipment (PPE) is available for them to wear. ${ }^{2}$ The US Food and Drug Administration issued an Emergency Use Authorization for 2 messenger RNA COVID-19 vaccines. Although these vaccines have not been tested in pregnant women, ACOG recommends that COVID-19 vaccines not be withheld from pregnant women who fulfill the criteria for vaccination; pregnant women who decline vaccination should be supported in their decision. ${ }^{12}$ In dermatology, telemedicine is an effective alternative to face-to-face visits, reducing the risk of transmitting SARS-CoV-2 to physicians and patients.

Ideally, pregnant dermatology attending physicians and residents can continue to provide care through teledermatology. They also can continue to provide in-person care, if they choose to; however, higher-risk procedures should be avoided. ${ }^{12}$ In dermatology, that might include ablative laser procedures to the face, prolonged surgery, such as hair transplantation, and intraoral or intranasal procedures. Alternatively, pregnant dermatology residents can be allocated to clinical rotations in which face-to-face contact with patients is not required such as dermatopathology and a research rotation. Likewise, telework options can be encouraged for other pregnant members of dermatology teams, including front-desk staff, nurses, medical assistants, and remaining ancillary staff.

\section{Guidance on Face Masks for Pregnant HCWs}

Universal masking of HCWs has been shown to reduce the rate of health care-related acquisition of SARSCoV-2. ${ }^{13}$ However, extended use or reuse of N95 respirators might contribute to SARS-CoV-2 transmission. ${ }^{14}$ The
American College of Obstetricians and Gynecologists recommends that all HCWs wear a face mask at all times while working in a health care facility, even if patients are wearing a face covering or face mask. ${ }^{2}$ Based on CDC guidelines, ${ }^{15} \mathrm{HCWs}$ in regions where community transmission is moderate or substantial should wear eye protection in addition to a face mask, and they should wear an N95, N95-equivalent, or higher-level respirator instead of a face mask when performing aerosolgenerating procedures and surgical procedures. If working in a patient-facing role caring for patients with suspected or confirmed COVID-19, HCWs should wear an N95, N95-equivalent, or higher-level respirator; gown; gloves; and eye protection (goggles or a disposable face shield). ${ }^{15}$

\section{Final Thoughts}

COVID-19 has brought about acute and likely permanent changes to the US health care system. Dermatologists are integral members of that system and are essential to the treatment of patients with skin, hair, and nail disorders. Pregnant dermatologists and residents should refrain from patient-facing roles when feasible; however, when all recommended PPE are available, they may continue to work in patient-facing roles until they give birth if they desire to do so. Alternatively, teledermatology and non-face-to-face rotations should be encouraged. Higher-risk and aerosolgenerating procedures are of particular concern regarding the risk for transmitting SARS-CoV-2 and should be avoided. Correct and universal use of PPE is paramount; when all recommended PPE is not available, pregnant HCWs should avoid exposure to patients with suspected or confirmed COVID-19. These recommendations will help safeguard pregnant members of dermatology teams during the COVID-19 pandemic while maximizing patient care.

\section{REFERENCES}

1. Rashidi Fakari F, Simbar M. Coronavirus pandemic and worries during pregnancy; a letter to editor. Arch Acad Emerg Med. 2020;8:E21.

2. The American College of Obstetricians and Gynecologists. COVID-19 FAQs for obstetrician-gynecologists, obstetrics. 2020. Accessed April 21, 2021. https://www.acog.org/clinical-information/physician-faqs/covid19-faqs-for-ob-gyns-obstetrics

3. Schwartz DA, Graham AL. Potential maternal and infant outcomes from (Wuhan) coronavirus 2019-nCoV infecting pregnant women: lessons from SARS, MERS, and other human coronavirus infections. Viruses. 2020;12:194. doi:10.3390/v12020194

4. Yan J, Guo J, Fan C, et al. Coronavirus disease 2019 in pregnant women: a report based on 116 cases. Am J Obstet Gynecol. 2020;223:111.e1-111. e14. doi:10.1016/j.ajog.2020.04.014

5. Zambrano LD, Ellington S, Strid P, et al; CDC COVID-19 Response Pregnancy and Infant Linked Outcomes Team. Update: characteristics of symptomatic women of reproductive age with laboratoryconfirmed SARS-CoV-2 infection by pregnancy status-United States, January 22-October 3, 2020. MMWR Morb Mortal Wkly Rep. 2020;69:1641-1647. doi:10.15585/mmwr.mm6944e3

6. Dubey P, Reddy SY, Manuel S, et al. Maternal and neonatal characteristics and outcomes among COVID-19 infected women: an updated systematic review and meta-analysis. Eur J Obstet Gynecol Reprod Biol. 2020;252:490-501. doi:10.1016/j.ejogrb.2020.07.034

7. Kotlyar AM, Grechukhina O, Chen A, et al. Vertical transmission of coronavirus disease 2019: a systematic review and meta-analysis. Am J Obstet Gynecol. 2020;224:35-53.e3. doi:10.1016/j.ajog.2020.07.049 
8. Mitchell T, MacDonald JW, Srinouanpranchanh S, et al. Evidence of cardiac involvement in the fetal inflammatory response syndrome: disruption of gene networks programming cardiac development in nonhuman primates. Am J Obstet Gynecol. 2018;218:438.e1-438.e16. doi:10.1016/j.ajog.2018.01.009

9. Ellington S, Strid P, Tong VT, et al. Characteristics of women of reproductive age with laboratory-confirmed SARS-CoV-2 infection by pregnancy status-United States, January 22-June 7, 2020. MMWR Morb Mortal Wkly Rep. 2020;69:769-775. doi:10.15585/mmwr .mm6925a1

10. Panagiotakopoulos L, Myers TR, Gee J, et al. SARS-CoV-2 infection among hospitalized pregnant women: reasons for admission and pregnancy characteristics - eight U.S. health care centers, March 1-May 30, 2020. 2020. doi:10.15585/mmwr.mm6938e2

11. Knight M, Bunch K, Vousden N, et al; UK Obstetric Surveillance System SARS-CoV-2 Infection in Pregnancy Collaborative Group. Characteristics and outcomes of pregnant women admitted to hospital with confirmed SARS-CoV-2 infection in UK: national population based cohort study. BMJ. 2020;369:m2107. doi:10.1136/bmj.m2107
12. The American College of Obstetricians and Gynecologists. Vaccinating pregnant and lactating patients against COVID-19. December 2020. Updated March 24, 2021. Accessed April 28, 2021. https://www.acog .org/clinical/clinical-guidance/practice-advisory/articles/2020/12 /vaccinating-pregnant-and-lactating-patients-against-covid-19

13. Seidelman JL, Lewis SS, Advani SD, et al. Universal masking is an effective strategy to flatten the severe acute respiratory coronavirus virus 2 (SARS-CoV-2) healthcare worker epidemiologic curve. Infect Control Hosp Epidemiol. 2020;41:1466-1467. doi:10.1017/ice.2020.313

14. Degesys NF, Wang RC, Kwan E, et al. Correlation between N95 extended use and reuse and fit failure in an emergency department. JAMA. 2020;324:94-96. doi:10.1001/jama.2020.9843

15. Centers for Disease Control and Prevention. Interim infection prevention and control recommendations for healthcare personnel during the coronavirus disease 2019 (COVID-19) pandemic 2020. Updated February 23, 2021. Accessed April 21, 2021. https://www.cdc.gov/coronavirus/2019-ncov/hcp/infection-control -recommendations.html?CDC_AA_refVal=https $\% 3 \mathrm{~A} \% 2 \mathrm{~F} \% 2 \mathrm{Fwww} . \mathrm{cdc}$ .gov\%2Fcoronavirus $\% 2$ F2019-ncov $\% 2$ Finfection-control $\% 2$ Fcontrol -recommendations.html 